\title{
Hemodynamic analysis of carotid artery after endarterectomy: a preliminary and quantitative imaging study based on computational fluid dynamics and magnetic resonance angiography
}

\author{
Yuanyuan Dai ${ }^{1,2}$, Peng Lv ${ }^{1}$, Ashkan Javadzadegan ${ }^{2}$, Xiao Tang ${ }^{3}$, Yi Qian ${ }^{2}$, Jiang Lin ${ }^{1}$ \\ ${ }^{1}$ Department of Radiology, Zhongshan Hospital of Fudan University and Shanghai Institute of Medical Imaging, Shanghai 200032, China; \\ ${ }^{2}$ Department of Biomedical Sciences, Faculty of Medicine and Health Sciences, Macquarie University, Sydney, Australia; ${ }^{3}$ Department of Vascular \\ Surgery, Zhongshan Hospital of Fudan University, Shanghai 200032, China
}

Correspondence to: Jiang Lin. Department of Radiology, Zhongshan Hospital of Fudan University and Shanghai Institute of Medical Imaging, Shanghai 200032, China. Email: lin.jiang@zs-hospital.sh.cn; Yi Qian. Department of Biomedical Sciences, Faculty of Medicine and Health Sciences, Macquarie University, Sydney, Australia. Email: yi.qian@mq.edu.au.

Background: The carotid blood flow following carotid endarterectomy (CEA) is not fully understood. Computational fluid dynamics (CFD) is a promising method to study blood flow. This study is to investigate local hemodynamic characteristics after CEA via the use of unenhanced magnetic resonance angiography (MRA) and CFD.

Methods: Eight carotid arteries with atherosclerosis and sixteen normal carotid arteries were included in this study. Time-of-flight (TOF) and phase contrast (PC) MRA were applied for the measurement of threedimensional artery geometries and velocity profile under CFD simulation. The hemodynamic parameters of the proximal internal carotid artery (ICA) including velocity, ICA/common carotid artery (CCA) velocity ratio, mean, maximum, minimum and gradient of wall shear stress $\left(\mathrm{WSS}_{\text {mean }}, \mathrm{WSS}_{\max }\right.$, $\mathrm{WSS}_{\min }$ and WSSG) were calculated before and after CEA. Morphologic characteristics of the carotid including bifurcation angle, tortuosity and planarity were also analyzed.

Results: Compared with pre-CEA, there was a significant reduction in post-CEA velocity, WSS $_{\max }$, $\mathrm{WSS}_{\text {mean }}$, and WSSG, by $87.24 \% \pm 13.38 \%, 86.86 \% \pm 14.97 \%, 57.32 \% \pm 56.71 \%$ and $69.74 \% \pm 37.03 \%$ respectively, whereas $W_{\text {min }}$ was almost unchanged. ICA/ CCA velocity ratios increased significantly after CEA. We also found that the post-CEA flow conditions were positively remodelled to approximate the conditions in normal arteries. The correlation between PC-MRA and CFD was excellent for the measurement of maximum velocity at the external carotid artery $(\mathrm{r}=0.846)$.

Conclusions: Our preliminary results indicated that major flow dynamics were restored shortly following CEA, and CFD based on MRA measurements could be useful for quantitative evaluation of hemodynamic outcomes after CEA.

Keywords: Carotid atherosclerosis; wall shear stress (WSS); computational fluid dynamics (CFD); magnetic resonance angiography (MRA)

Submitted Mar 07, 2018. Accepted for publication Apr 24, 2018.

doi: 10.21037/qims.2018.05.02

View this article at: http://dx.doi.org/10.21037/qims.2018.05.02 


\section{Introduction}

Carotid atherosclerosis is a major cause of stroke and transient ischemic attack. For patients with carotid atherosclerosis, carotid endarterectomy (CEA) or the surgical removal of the plaque is recommended as an effective method to reduce the risk of stroke (1), with the morphological correction of carotid stenosis. The effectiveness of CEA is largely evaluated via the measurement of luminal changes on imaging examinations or by reductions in cerebrovascular events during follow-up assessments. However, until now there have been only a limited number of studies focused on the evaluation of hemodynamic recovery following CEA.

Wall shear stress (WSS), a force of blood flow against the vessel wall, plays a critical role in the development of atherosclerotic plaque. Low and oscillator WSS are both linked with the initiation and progress of the atherosclerosis plaque (2), while high WSS may be associated with plaque vulnerability (3). Schirmer et al. showed that WSS began to approach normal levels following carotid stenting (4). Uemiya et al. reported that local hemodynamics altered after endarterectomy and stenting (5). Whether or not hemodynamics can be restored after CEA remains poorly understood (6). Furthermore, in order to better understand the mechanisms of restenosis after revascularization, it is also critical to study and monitor the local hemodynamic alterations after intervention.

Computational fluids dynamics (CFD) is an accepted method for in vivo hemodynamic analysis and has been extensively used in cardiovascular studies. CFD techniques can be utilized to simulate blood flows based on patient specific three-dimensional (3D) geometry derived from medical imaging. A number of studies have proved that the CFD method is a powerful and reliable tool for the hemodynamic analysis of carotid stenosis $(7,8)$. Recently, Guerciotti et al. used CFD to investigate the hemodynamic effects of CEA on carotid bifurcation, however, a normal control was not available in that study for comparison (9).

Time-of-flight magnetic resonance angiography (TOFMRA) is an unenhanced luminal angiography technique commonly used for morphological evaluation of carotid stenosis in clinical practice, especially in patients who have a risk of adverse reactions to contrast media or in those with impaired renal function (10). Phase contrast magnetic resonance angiography (PC-MRA) is another unenhanced MRA modality that provides in vivo blood velocity measurements (8). In this study, we employed CFD methods based on TOF- and PC-MRA measurements to quantitatively analyze carotid flows both before and after
CEA, with normal carotid arteries as a control group for comparison.

\section{Methods}

\section{Subjects}

Eight patients ( 7 males, 1 female; age range, $51-75$ years; mean age $\pm \mathrm{SD}, 65.5 \pm 7.8$ years) diagnosed with carotid atherosclerosis, who underwent CEA were recruited. The degree of stenosis within all eight arteries was over $70 \%$, with mean stenosis $84.21 \% \pm 8.87 \%$. Three of the treated arteries were right-sided, whilst five were left-sided. The stenosis was located at the proximal internal carotid artery (ICA) or carotid bulb. All operations were carried out by the same vascular surgeon (Xiao Tang). Ten volunteers with 16 normal carotid arteries (9 males, 1 female; age range, $41-76$ years; mean age $\pm S D, 48.2 \pm 10.9$ years) were enrolled as a control group in this study. The study was approved of by the local institutional ethic board, with informed consent obtained from all participants.

\section{MRA protocols and analysis}

All patients with carotid atherosclerosis underwent MRA twice, one week prior to and 15 days post CEA operation. MRA was performed using a 3.0-T magnetic resonance imaging scanner (Magnetom Verio; Siemens, Erlangen, Germany) with an 8-channel carotid artery coil (Chenguang Technology, Shanghai, China).

MRA sequences included 3D TOF-MRA and a twodimensional (2D) time-resolved PC-MRA sequence triggered by an electrocardiogram gating. The parameters for TOF-MRA were: repetition time/echo time (TR/TE) $26 / 3.23 \mathrm{~ms}$, flip angle $25^{\circ}$, thickness $2 \mathrm{~mm}$, field-of-view (FOV) $160 \mathrm{~mm} \times 160 \mathrm{~mm}$, matrix 256×256, and acquisition time 2.33 minutes. The longitudinal coverage of TOF-MRA was approximately $7 \mathrm{~cm}$ including the distal common carotid artery (CCA), carotid bifurcation, the proximal ICA and the proximal external carotid artery (ECA). The parameters for PC-MRA sequence were as following: TR/TE 13/4 ms, slice thickness $1.0 \mathrm{~mm}$, FOV $140 \mathrm{~mm} \times 140 \mathrm{~mm}$, matrix $256 \times 256$. The maximum velocity was targeted in this study, thus the encoded velocity was set at a range between 100 $200 \mathrm{~cm} / \mathrm{s}(11,12)$.

\section{MRA processing}

The measurement of PC-MRA was performed independently 
by two experienced radiologists (Yuanyuan Dai, Peng Lv), with the mean values from the two measurements recorded.

2D PC-MRA was applied to obtain the velocities in the vicinity of the carotid bifurcation. Velocity was measured at different points at the CCA, ICA and ECA respectively. These measurement points were $2 \mathrm{~cm}$ proximal to the carotid bifurcation at CCA and $2 \mathrm{~cm}$ distal to stenosis at ICA, or about $3 \mathrm{~cm}$ distal to the carotid bifurcation of the normal ICA. On ECA, the measurement point was $1-2 \mathrm{~cm}$ distal to the bifurcation where no major branches arose, with measurement planes orthogonal to the long axis of the target vessels.

Two sets of cross-sectional images including magnitude images and phase images were obtained from PC-MRA. Region of interest (ROI) was drawn on the magnitude image, with velocity information extracted from the phase image corresponding to the ROI on the magnitude image. Mean velocity from the cardiac circle was recorded at each measurement point.

\section{Computational fluid dynamics (CFD)}

The 3D geometry of carotid bifurcation was reconstructed based on 3D TOF-MRA data using commercial software Mimics 16.0 (Materialise Company, Leuven, Belgium). The $3 \mathrm{D}$ reconstructed geometry was then imported as a stereolithography (STL) file into ICEM 15.0 (ANSYS, Lebanon, NH, USA) for CFD mesh generation. A boundary layer mesh with a growth factor of 1.2 was used to improve the accuracy for the measurement of nearwall hemodynamic parameters. The total mesh size ranged from 1.6 to 3 million hexahedral and prism elements. To minimise the effects of boundary conditions on flow behaviour, the vessel was extended at the inlet and outlet to a length that is 20 times of the vessel diameter. All CFD simulations were normally converged and reached the criteria of residual target 0.0001 .

Blood was considered as a non-Newtonian fluid with a density of $1,050 \mathrm{~kg} / \mathrm{m}^{3}$ and a viscosity of $0.0035 \mathrm{~Pa} \cdot \mathrm{s}(5)$. CFX 15.0 (ANSYS, Lebanon, NH, USA) was implemented for fluid-flow modelling, with the flow assumed to be steady and incompressible (13). The vessel walls were assumed to be rigid with non-slip boundary conditions. The velocity at CCA measured via PC-MRA was averaged over a cardiac cycle and used as the inlet boundary condition. As the stenotic segment was located at ICA, measured average velocity at the ICA was imposed as the outlet boundary condition, with a zero pressure condition applied at the outlet of the ECA.

Flow velocity ratios of ICA to CCA were calculated and compared among pre-, post-CEA and normal carotid arteries. The velocity, WSS in mean $\left(\mathrm{WSS}_{\text {mean }}\right)$, maximum $\left(\mathrm{WSS}_{\text {max }}\right)$, minimum $\left(\mathrm{WSS}_{\min }\right)$, as well as WSS gradient (WSSG) were calculated both pre- and post-CEA, and compared to those of the control group. Velocity was defined as the maximum velocity at the most stenotic area within ICA pre-CEA. At post-CEA arteries, the velocity was measured at the same locations as pre-CEA, and for health arteries the velocity was calculated at ICA about $1 \mathrm{~cm}$ distal to the bifurcation. The $\mathrm{WSS}_{\text {mean }}, \mathrm{WSS}_{\max }, \mathrm{WSS}_{\min }$ and WSSG of ICA were calculated at the same locations respectively.

\section{Morphology of bifurcation}

Morphologic characteristics were analyzed using Solid Edge ST9 (Siemens PLM Software, Plano, Texas, USA). Geometric factors including bifurcation angle, tortuosity and planarity angle were analyzed in this study. These geometric factors were defined using the method described in our previous study (5). Tortuosity was defined using a formula $\mathrm{L} / \mathrm{D}-1$, where $\mathrm{L}$ is the length of the center line from CCA to ICA end point, and D is the straight line distance between these two points. Bifurcation angle was defined as the angles between the center lines of ICA and ECA when viewed from the front. The planarity angle was defined as the angle between ECA and ICA in the lateral view.

\section{Statistical analysis}

The demographic data from the patients and healthy subjects were compared by using chi-square test (categorized variables) and independent $t$-test (continuous variables). Inter-observer reproducibility with respect to the velocity measured by PC-MRA was analyzed using interclass correlation coefficient (ICC). The results of both hemodynamic and morphological characteristics including velocity, velocity ratio of ICA/CCA, WSS $_{\max }$, $\mathrm{WSS}_{\text {mean }}, \mathrm{WSS}_{\min }$, WSSG, bifurcation angle, tortuosity and planarity both before and after CEA were compared using the non-parametric paired test. The hemodynamic and morphological parameters of pre- and post-CEA arteries, as well as of healthy arteries were compared via the use of independent $t$-tests. Pearson correlation was used to examine the correlation between PC-MRA and CFD simulation in the measurement of velocity at the ECA. The coefficient ratio $r>0.80$ was considered as an excellent 
correlation (14). The mean value \pm standard deviation (SD) was computed for all data. Two-sided tests were performed and $\mathrm{P}<0.05$ was considered statistically significant. SPSS 22.0 statistical software (IBM, Chicago, IL, USA) was used for statistical analysis.

\section{Results}

The demographic data from eight patients with carotid atherosclerosis and ten volunteers were listed and compared in Table 1. The inter-observer reliability of the velocity measurement obtained from PC-MRA was excellent. (PreCEA CCA, ICC $=0.970$; pre-CEA ICA, ICC $=0.859$; preCEA ECA, ICC $=0.976$; post-CEA CCA, ICC $=0.988$; post-CEA ICA, ICC $=0.938$; post-CEA ECA, ICC $=0.944$; normal CCA, ICC $=0.979$; normal ICA, ICA $=0.946$; normal ECA, ICC $=0.952$ ).

An illustration of 3D TOF-MRA and the velocities measured by PC-MRA was shown in Figures 1,2. The results of CFD and statistical analysis were shown in

Table 1 Baseline characteristics of the patients with carotid atherosclerosis and the controls

\begin{tabular}{lccc}
\hline Variable & CEA patients $(\mathrm{n}=8)$ & Control group $(\mathrm{n}=10)$ & $\mathrm{P}$ value \\
\hline Age (years) & $65.5 \pm 7.8$ & $48.2 \pm 10.9$ & 0.002 \\
Gender & 7 males & 9 males & 0.867 \\
Smoker (ever) & 5 & 0 & 0.005 \\
Diabetes & 2 & 1 & 0.094 \\
Hypertension & 6 & $119.0 \pm 8.8$ & 0.003 \\
Systolic blood pressure (mmHg) & $156.3 \pm 22.0$ & $78.6 \pm 8.3$ & $<0.001$ \\
Diastolic blood pressure $(\mathrm{mmHg})$ & $101.9 \pm 13.1$ & 0 & $<0.001$ \\
Hyperlipidemia & 1 & $1.13 \pm 0.27$ & 0.25 \\
HDL-cholesterol (mmol/L) & $1.06 \pm 0.14$ & $2.97 \pm 0.88$ & 0.166 \\
Total cholesterol (mmol/L) & $3.58 \pm 0.89$ & 0 & - \\
Statins user & 0 & 0.487 \\
\hline
\end{tabular}

$\mathrm{n}$, indicates the number of subjects. CEA, carotid endarterectomy; HDL, high density lipoprotein.
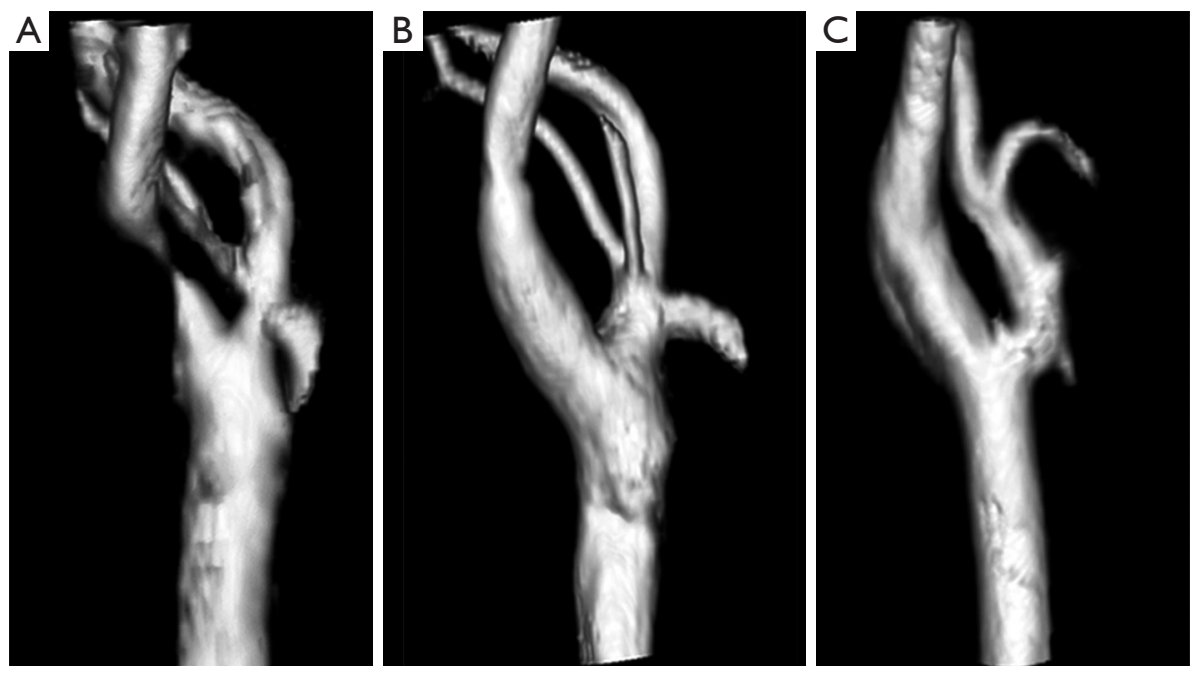

Figure 13 D TOF-MRA images generated from pre-CEA (A), post-CEA (B) and normal carotids (C). Pre- and post-CEA carotids are from a 59-year-old male with severe stenosis at the proximal internal carotid artery 3 days before and 5 days after CEA. The normal carotid is from a 48-year-old healthy male. TOF-MRA, time-of-flight magnetic resonance angiography; CEA, carotid endarterectomy. 

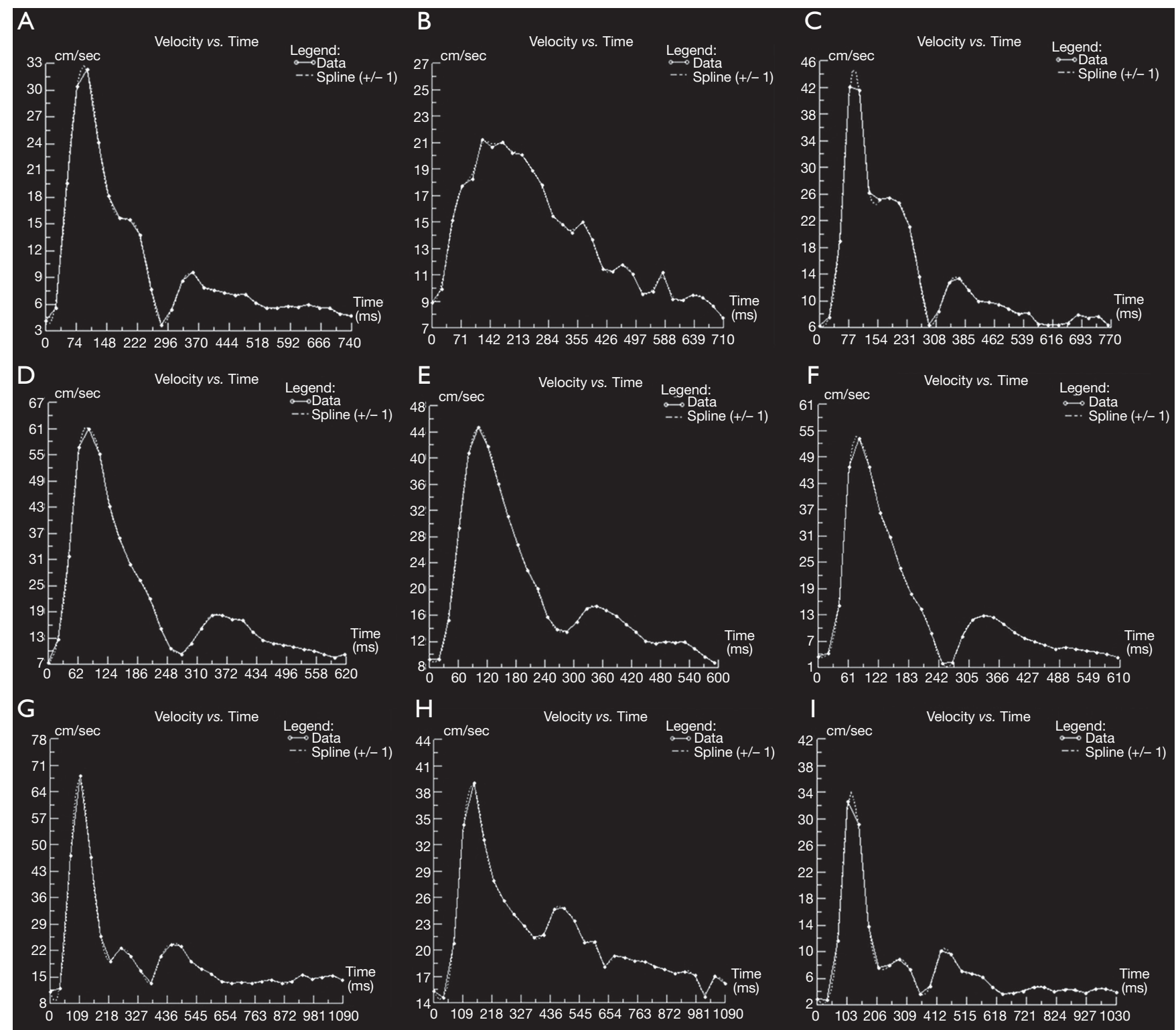

Figure 2 Velocities measured by PC-MRA. (A,B,C) Show velocities from CCA (A), ICA (B) and ECA (C) of a pre-CEA carotid (the same artery as Figure 1A). The velocities of the post-CEA carotid (the same artery as Figure 1B) in CCA (D), ICA (E) and ECA (F) are shown. The velocities of a normal carotid (the same artery as Figure 1C) measured in CCA (G), ICA (H) and ECA (I) are demonstrated. PC-MRA, phase contrast magnetic resonance angiography; CCA, common carotid artery; ICA, internal carotid artery; ECA, external carotid artery; CEA, carotid endarterectomy.

Figure 3 and summarized in Table 2. The results showed significant differences before and after CEA in the velocity, ICA/CCA velocity ratio, WSS $_{\max }$, and WSSG. However, no significant difference was found in $\mathrm{WSS}_{\min }$. When comparing hemodynamic results between pre-CEA and normal arteries, the flow parameters within pre-CEA were noticeably higher than those within normal arteries including the velocity, $\mathrm{WSS}_{\max }, \mathrm{WSS}_{\text {mean }}$ and WSSG. The ICA/CCA velocity ratios before CEA were lower than those after CEA. However, there was no significant difference in $\mathrm{WSS}_{\min }$. The result indicated that $\mathrm{WSS}_{\text {min }}$ may not be a sensitive parameter to observe changes within the ICA artery. Furthermore, we found that flow characteristics have been positively recovered after CEA surgery, and that there 
A

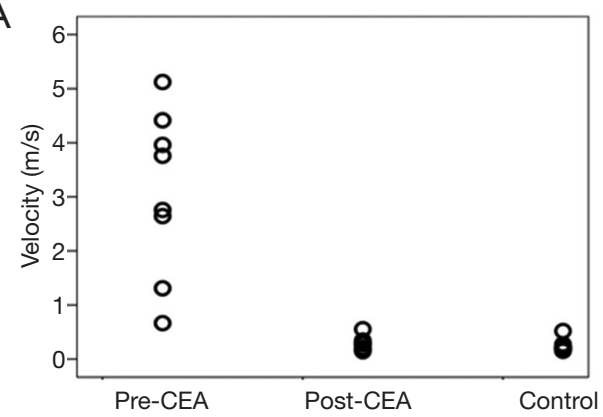

C

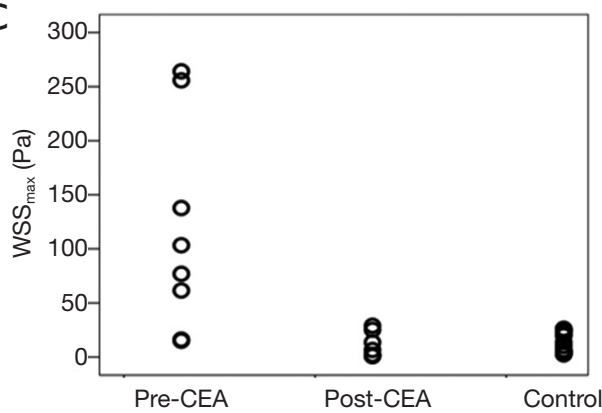

E

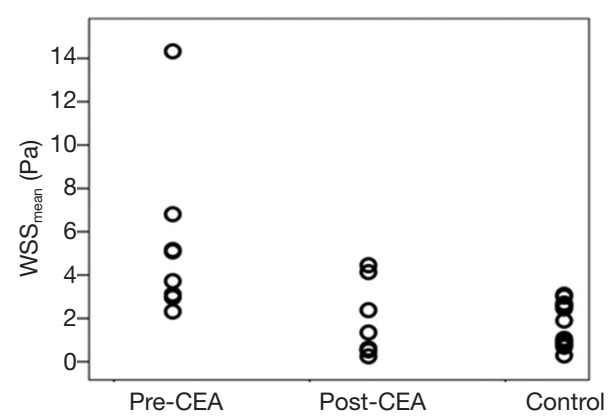

B

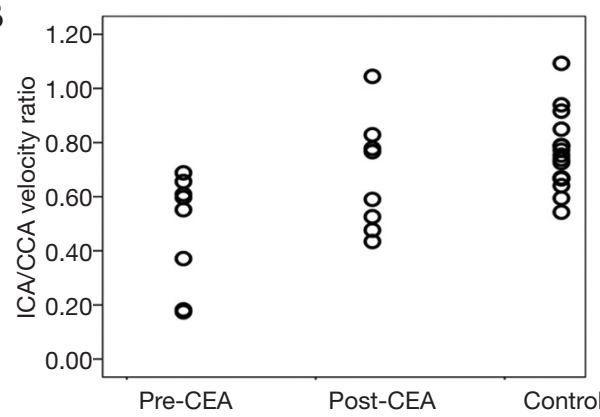

D

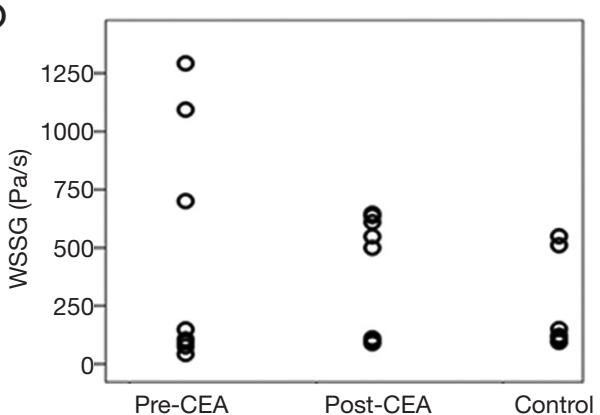

$\mathrm{F}$

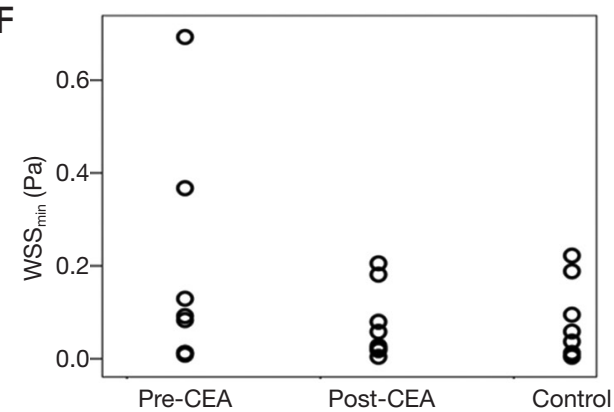

Figure 3 Scatter plots show the hemodynamic parameters of velocity (A), ICA/CCA velocity ratio (B), WSS max $_{\text {(C), WSSG (D), WSS }}$ mean $($ E) and $\mathrm{WSS}_{\min }(\mathrm{F})$ from eight stenotic carotids before and after CEA and sixteen normal carotids (control). ICA, internal carotid artery; CCA, common carotid artery; CEA, carotid endarterectomy; WSS, wall shear stress.

was no significant flow difference between post-CEA and normal carotids in velocity and ICA/CCA velocity ratio, $\mathrm{WSS}_{\text {max }}, \mathrm{WSS}_{\text {mean }}, \mathrm{WSS}_{\text {min }}$, and WSSG. WSS distributions after surgery were recovered to be close to those of the normal carotids, whilst a large difference was found in WSS distributions between pre-CEA carotids and those control ones (Figure 4).

The bifurcation angle, tortuosity and planarity in preCEA, post-CEA and normal arteries were analyzed and compared (Table 3). No significant difference was found in bifurcation angle, tortuosity and planarity between pre-CEA and post-CEA arteries. However, the bifurcation angles of pre-CEA carotids were smaller than those of normal carotids. In addition, we found that all cases had an increased bifurcation angle after surgery with the exception of one case. Compared with pre-CEA results, four post-CEA cases showed increased bifurcation tortuosity, while the other four decreased tortuosity. Four cases had larger planarity whilst the other cases had smaller one. We also observed an interesting phenomenon that post-surgery arteries which had relatively smaller tortuosities created relatively higher WSS values at carotid bifurcation (Figure 5).

Pearson correlation coefficient showed excellent agreement $(\mathrm{r}=0.846, \mathrm{P}<0.001)$ between the results of $\mathrm{CFD}$ and PC-MRA in the measurement of velocity at the ECA (Figure 6). 
Table 2 Flow comparison between pre-, post-CEA and normal carotids

\begin{tabular}{|c|c|c|c|c|c|c|}
\hline Flow parameters & Pre-CEA $(n=8)$ & Post-CEA $(n=8)$ & $\begin{array}{l}\text { Normal carotids } \\
\qquad(n=16)\end{array}$ & \multicolumn{3}{|c|}{$P$ value } \\
\hline ICA/CC velocity ratio & $0.48 \pm 0.21$ & $0.68 \pm 0.21$ & $0.76 \pm 0.14$ & 0.025 & 0.001 & 0.283 \\
\hline WSSG (Pa/s) & $3,199.33 \pm 3,405.31$ & $443.79 \pm 511.41$ & $331.78 \pm 239.83$ & 0.012 & 0.002 & 0.393 \\
\hline $\mathrm{WSS}_{\min }(\mathrm{Pa})$ & $0.02 \pm 0.02$ & $0.2 \pm 0.2$ & $0.08 \pm 0.08$ & 0.575 & 0.050 & 0.897 \\
\hline
\end{tabular}

$\mathrm{n}$, indicates the number of subjects. CEA, carotid endarterectomy; ICA, internal carotid artery; CCA, common carotid artery.
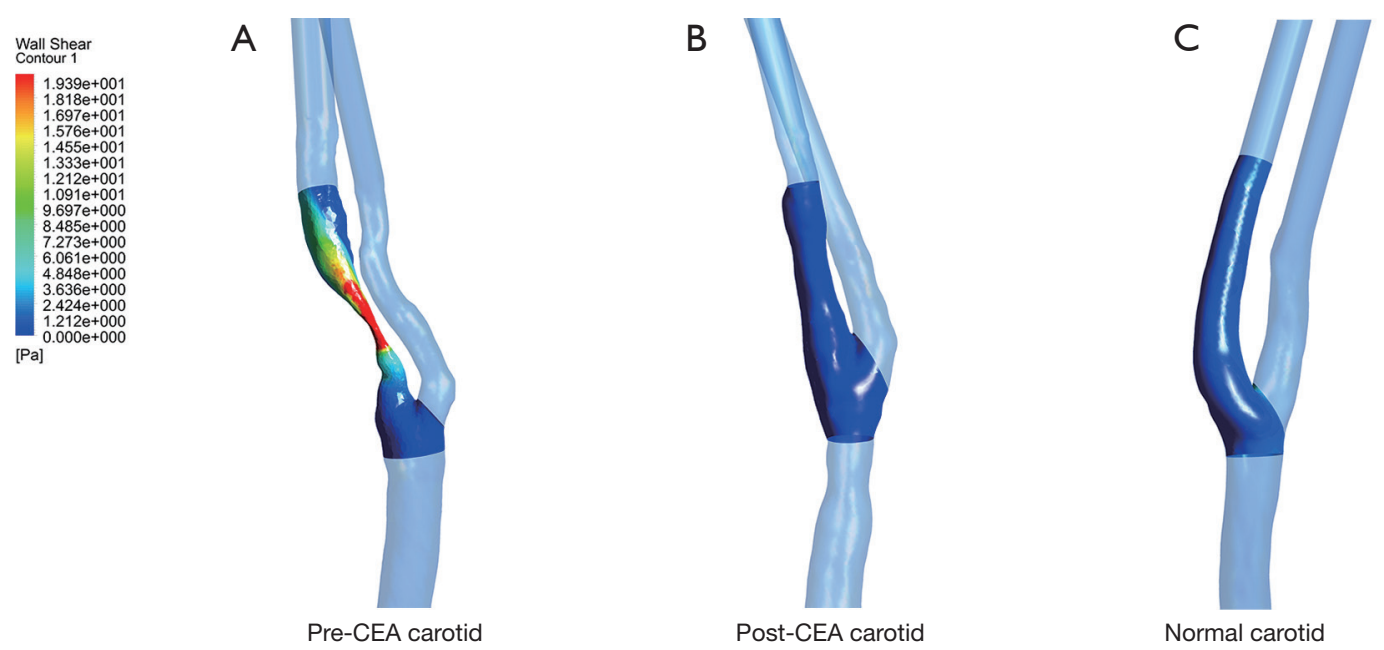

Figure 4 Wall shear stress map showed that the distribution of wall shear stress was demonstrated on a pre-CEA (A), post-CEA (B) and normal carotid (C). Wall shear stress increases significantly at stenotic area of pre-CEA carotid. In contrast, the wall shear stress profile after CEA from the same patient is similar to that of a normal carotid. CEA, carotid endarterectomy.

\section{Discussion}

This preliminary study showed that velocity, ICA/CCA velocity ratio, $\mathrm{WSS}_{\max }, \mathrm{WSS}_{\text {mean }}$ and WSSG significantly altered after CEA. With normal arteries as a control group, these main hemodynamic parameters of post-CEA arteries were recovered to those normal levels. In addition, we found that bifurcation angle alternations before and after CEA had connections with hemodynamic changes. Based on our results, we can draw a conclusion that the application of unenhanced MRA combined with CFD could provide comprehensive understanding for both flow dynamic and morphological alterations after CEA. The combination of unenhanced MRA and CFD method may greatly facilitate the blood flow follow-up studies after surgery, as it is noninvasive and safe.

Four-dimensional (4D) flow MRI is able to measure both velocity and WSS at the carotid bifurcation $(8,15)$. Harloff et al. (6) compared flow parameters before and after CEA via the use of 4D flow MRI and found that regional WSS decreased after CEA surgery. An earlier study explored similar techniques and showed that the distribution of WSS within stenotic arteries differed greatly from normal arteries, and that the flow distribution in post-operative arteries returned to values that closely resembled pre-operative conditions (16). Compared with CFD, limitations in 4D flow MRI such as lower spatial and temporal resolution, and relatively poorer delineations in 
Table 3 Comparisons of morphologic characteristics between pre-, post-CEA and normal carotids

\begin{tabular}{|c|c|c|c|c|c|c|}
\hline Morphologic parameters & Pre-CEA $(n=8)$ & Post-CEA $(n=8)$ & $\begin{array}{l}\text { Normal carotids } \\
\qquad(n=16)\end{array}$ & \multicolumn{3}{|c|}{$P$ value } \\
\hline Tortuosity & $0.08 \pm 0.06$ & $0.06 \pm 0.04$ & $0.06 \pm 0.03$ & 0.573 & 0.594 & 0.655 \\
\hline
\end{tabular}

$\mathrm{n}$, indicates the number of subjects. CEA, carotid endarterectomy.
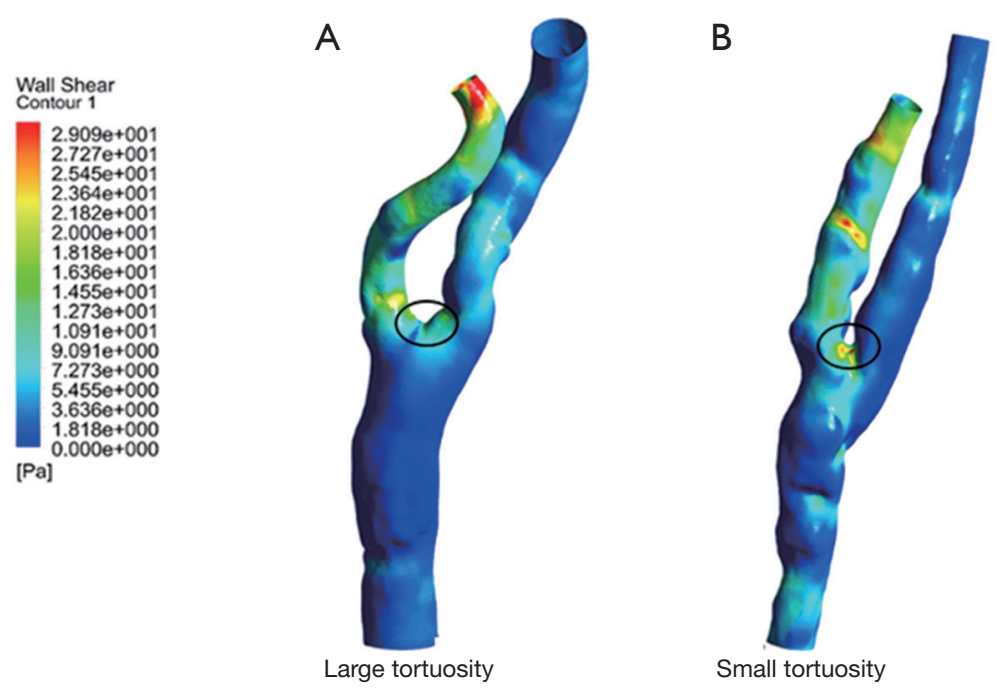

Figure 5 Wall shear stress map demonstrated that (A) the carotid with large tortuosity showed relatively low wall shear stress at bifurcation area (circle), while the carotid with small tortuosity showed relatively high wall shear stress at bifurcation (B).

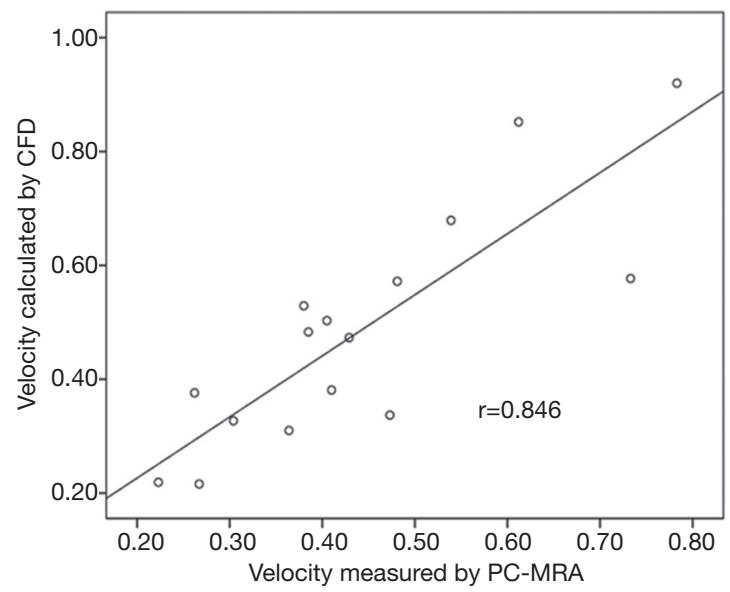

Figure 6 There is an excellent agreement between CFD and PCMRA in measurement of the velocity at external carotid artery. CFD, computational fluid dynamics; PC-MRA, phase contrast magnetic resonance angiography. vessel surfaces may hamper the computation of absolute hemodynamic values $(13,17)$. Furthermore, 4D flow MRI measurements require relatively long acquisition times depending on the patient's heart rate $(13,17)$ whilst special software that are not yet widely available, may limit its clinical application (18).

CFD has been applied in the hemodynamic assessment of blood flow. Accurate CFD simulation depends on patient-specific anatomy and flow boundary conditions. In real clinical scenarios, however, flow boundary conditions are not always available (19). As a result, the boundary conditions for CFD simulation were typically assumed with statistic or mean values obtained from the literature $(7,20)$. This may create inaccuracies on an individual basis, especially at areas with complex geometry, which may lead to errors in the simulation of outcomes. In this study, however, we employed flow results from PC-MRA to assess 
individual flow boundary conditions. With this patientspecific measurement, our results and comparison studies increase in reliability and clinical relevance.

Using CFD technique, Guerciotti et al. (9) showed that both peak velocity and maximum WSS decreased significantly after CEA in patients with severe stenosis. However, since the study lacked a comparison with normal artery hemodynamics, the extent of the hemodynamic recovery after CEA could not be reliably appreciated. Due to localized dilatation at the carotid bifurcation and insufficient understanding of the flow dynamics following CEA, the establishment of a normal control is paramount for the accurate interpretation of CFD measurements. In this study, we found that both the magnitude and distribution of WSS at the bifurcation after CEA were similar to those of controls. We found that both carotid geometry and flow were recovered shortly after CEA, with local post-operative dilatation seemingly having little effect on the flow.

The recurrence rate of stenosis after CEA was approximately $4.1-5.8 \%$ (15). Since flow dynamics plays a critical role in the development and progression of atherosclerosis, its alteration may also lead to restenosis after revascularization surgery. CFD would be a valuable tool to inspect flow after CEA. However, a longer period of observation will be necessary to confirm carotid geometry remodelling and flow alternation which may lead to restenosis.

In order to inspect concordance between CFD simulations and PC-MRA measurements, we performed a correlation study for velocity measurements at the ECA. An excellent correlation was shown between PC-MRA measurements and CFD simulation. The small differences between the two measurements could be attributed to the errors from PC-MRA, caused by dephasing effects at the stenosis. However, a deviation of less than $10 \%$ by PC MRA was considered acceptable (8). Additionally, small arteries which branched off the ECA were not included in CFD modelling, another potential reason to explain the velocity differences between the two methods.

From the comparisons of morphologic characteristics, we observed the bifurcation angles of pre-surgery arteries were smaller than those normal ones. Although no significant difference was found in bifurcation angles between pre-CEA and post-CEA carotids, in seven arteries they increased after CEA. We believe that CEA could increase the bifurcation angle in most carotids with stenosis located at proximal ICA and carotid bulb. Our study also showed that no significant difference between tortuosity and planarity between preCEA and post-CEA arteries. It appeared that surgery might have different impacts on tortuosity and planarity of different carotids. Further studies with more cases are necessary to verify our observations.

Morphologic characteristics are thought to contribute to local hemodynamics. In our study, we found that the WSS differed between post-CEA cases with different tortuosities. In cases featuring small tortuosities, WSS values were higher at the bifurcation following surgery. As shown in a previous study, restenosis after carotid stenting occurred more frequently in arteries with smaller tortuosities (5). We speculate that carotids with small tortuosity might have a higher risk of restenosis after CEA, although follow-up observations are required to support this assumption. We suggest that morphological analysis could also provide extra information for follow-up assessment of carotid artery after surgical intervention.

There were some limitations in this study. The vessel wall surface was assumed to be rigid, and CFD simulation was performed as a steady flow. Although carotid flow is pulsatile, its vessel surface displacement is typically less than $10 \%$ (21). A previous study demonstrated that the rigid wall assumption had little effect on WSS calculation when the vessel wall displacement was small (22). Another limitation was that long-term follow-up was not available and the study was limited by a small number of cases. Although hemodynamic recovery after CEA was convincingly demonstrated through intra-individual and inter-individual comparison in this study, a large population of patients and a longer time of follow-up would be necessary to further observe its consequences.

\section{Conclusions}

For carotid arteries with severe stenosis, major flow dynamics at bifurcation was found to be restored shortly after CEA. CFD simulation based on unenhanced MRAs was able to effectively evaluate hemodynamic changes both before and after CEA. It is a promising technique in the assessment and follow-up of the patient-specific effectiveness of revascularization.

\section{Acknowledgements}

The authors thank Xi Zhao for the support of CFD processing. 


\section{Footnote}

Conflicts of Interest: The authors have no conflicts of interest to declare.

Ethical Statement: The study was approved of by the local institutional ethic board, with informed consent obtained from all participants.

\section{References}

1. Biller J, Feinberg WM, Castaldo JE, Whittemore AD, Harbaugh RE, Dempsey RJ, Caplan LR, Kresowik TF, Matchar DB, Toole JF, Easton JD, Adams HP Jr, Brass LM, Hobson RW 2nd, Brott TG, Sternau L. Guidelines for carotid endarterectomy: a statement for healthcare professionals from a Special Writing Group of the Stroke Council, American Heart Association. Circulation 1998;97:501-9.

2. Thim T, Hagensen MK, Horlyck A, Kim WY, Niemann AK, Thrysoe SA, Drouet L, Paaske WP, Botker HE, Falk E. Wall shear stress and local plaque development in stenosed carotid arteries of hypercholesterolemic minipigs. J Cardiovasc Dis Res 2012;3:76-83.

3. Tuenter A, Selwaness M, Arias Lorza A, Schuurbiers JCH, Speelman L, Cibis M, van der Lugt A, de Bruijne $M$, van der Steen AFW, Franco OH, Vernooij MW, Wentzel JJ. High shear stress relates to intraplaque haemorrhage in asymptomatic carotid plaques. Atherosclerosis 2016;251:348-54.

4. Schirmer CM, Malek AM. Patient based computational fluid dynamic characterization of carotid bifurcation stenosis before and after endovascular revascularization. J Neurointerv Surg 2012;4:448-54.

5. Uemiya N, Lee CJ, Ishihara S, Yamane F, Zhang Y, Qian $\mathrm{Y}$. Analysis of restenosis after carotid artery stenting: preliminary results using computational fluid dynamics based on three-dimensional angiography. J Clin Neurosci 2013;20:1582-7.

6. Harloff A, Berg S, Barker AJ, Schollhorn J, Schumacher M, Weiller C, Markl M. Wall shear stress distribution at the carotid bifurcation: influence of eversion carotid endarterectomy. Eur Radiol 2013;23:3361-9.

7. Gharahi H, Zambrano BA, Zhu DC, DeMarco JK, Baek S. Computational fluid dynamic simulation of human carotid artery bifurcation based on anatomy and volumetric blood flow rate measured with magnetic resonance imaging. Int J Adv Eng Sci Appl Math 2016;8:40-60.
8. Calderon-Arnulphi M, Amin-Hanjani S, Alaraj A, Zhao M, Du X, Ruland S, Zhou XJ, Thulborn KR, Charbel FT. In vivo evaluation of quantitative $M R$ angiography in a canine carotid artery stenosis model. AJNR Am J Neuroradiol 2011;32:1552-9.

9. Guerciotti B, Vergara C, Azzimonti L, Forzenigo L, Buora A, Biondetti P, Domanin M. Computational study of the fluid-dynamics in carotids before and after endarterectomy. J Biomech 2016;49:26-38.

10. Debrey SM, Yu H, Lynch JK, Lovblad KO, Wright VL, Janket SJ, Baird AE. Diagnostic accuracy of magnetic resonance angiography for internal carotid artery disease: a systematic review and meta-analysis. Stroke 2008;39:2237-48.

11. Harloff A, Zech T, Wegent F, Strecker C, Weiller C, Markl M. Comparison of blood flow velocity quantification by $4 \mathrm{D}$ flow MR imaging with ultrasound at the carotid bifurcation. AJNR Am J Neuroradiol 2013;34:1407-13.

12. Khodarahmi I. Comparing velocity and fluid shear stress in a stenotic phantom with steady flow: phase-contrast MRI, particle image velocimetry and computational fluid dynamics. MAGMA 2015;28:385-93.

13. Cibis M, Potters WV, Selwaness M, Gijsen FJ, Franco $\mathrm{OH}$, Arias Lorza AM, de Bruijne M, Hofman A, van der Lugt A, Nederveen AJ, Wentzel JJ. Relation between wall shear stress and carotid artery wall thickening MRI versus CFD. J Biomech 2016;49:735-41.

14. Kwee RM, Teule GJ, van Oostenbrugge RJ, Mess WH, Prins MH, van der Geest RJ, Ter Berg JW, Franke CL, Korten AG, Meems BJ, Hofman PA, van Engelshoven JM, Wildberger JE, Kooi ME. Multimodality imaging of carotid artery plaques: $18 \mathrm{~F}$-fluoro-2-deoxyglucose positron emission tomography, computed tomography, and magnetic resonance imaging. Stroke 2009;40:3718-24.

15. Kumar R, Batchelder A, Saratzis A, AbuRahma AF, Ringleb P, Lal BK, Mas JL, Steinbauer M, Naylor AR. Restenosis after Carotid Interventions and Its Relationship with Recurrent Ipsilateral Stroke: A Systematic Review and Meta-analysis. Eur J Vasc Endovasc Surg 2017;53:766-75.

16. Markl M, Wegent F, Zech T, Bauer S, Strecker C, Schumacher M, Weiller C, Hennig J, Harloff A. In vivo wall shear stress distribution in the carotid artery: effect of bifurcation geometry, internal carotid artery stenosis, and recanalization therapy. Circ Cardiovasc Imaging 2010;3:647-55.

17. Cibis M, Potters WV, Gijsen FJ, Marquering H, vanBavel E, van der Steen AF, Nederveen AJ, Wentzel JJ. Wall shear stress calculations based on 3D cine phase contrast MRI 
and computational fluid dynamics: a comparison study in healthy carotid arteries. NMR Biomed 2014;27:826-34.

18. Markl M, Schnell S, Wu C, Bollache E, Jarvis K, Barker AJ, Robinson JD, Rigsby CK. Advanced flow MRI: emerging techniques and applications. Clin Radiol 2016;71:779-95.

19. Dong J, Inthavong K, Tu J. Image-based computational hemodynamics evaluation of atherosclerotic carotid bifurcation models. Comput Biol Med 2013;43:1353-62.

20. Ohhara Y, Oshima M, Iwai T, Kitajima H, Yajima Y, Mitsudo K, Krdy A, Tohnai I. Investigation of blood flow

Cite this article as: Dai Y, Lv P, Javadzadegan A, Tang X, Qian Y, Lin J. Hemodynamic analysis of carotid artery after endarterectomy: a preliminary and quantitative imaging study based on computational fluid dynamics and magnetic resonance angiography. Quant Imaging Med Surg 2018;8(4):399-409. doi: 10.21037/qims.2018.05.02 in the external carotid artery and its branches with a new OD peripheral model. Biomed Eng Online 2016;15:16.

21. Cinthio M, Ahlgren AR, Bergkvist J, Jansson T, Persson HW, Lindstrom K. Longitudinal movements and resulting shear strain of the arterial wall. Am J Physiol Heart Circ Physiol 2006;291:H394-402.

22. Zhao SZ, Xu XY, Hughes AD, Thom SA, Stanton AV, Ariff B, Long Q. Blood flow and vessel mechanics in a physiologically realistic model of a human carotid arterial bifurcation. J Biomech 2000;33:975-84. 